\title{
LA CONJECTURE DE BAUM-CONNES POUR UN FEUILLETAGE SANS HOLONOMIE DE CODIMENSION UN SUR UNE VARIÉTÉ FERMÉE
}

\author{
MARTA MACHO STADLER
}

\begin{abstract}
In [C2], Baum-Connes state a conjecture for the $\mathrm{K}$-theory of $\mathrm{C}^{*}$-algebras of foliations. This conjecture has been proved by T. Natsume [N2] for $\mathrm{C}^{\infty}$-codimension one foliations without holonomy on a closed manifold. We propose here another proof of the conjecture for this class of foliations, more geometric and based on the existence of the Thom isomophism, proved by A. Connes in [C3]. The advantage of this approach is that the result will be valid for all $C^{0}$-foliations.
\end{abstract}

\section{0 . Introduction}

Baum-Connes énoncent dans [C2] une conjecture pour la $\mathrm{K}$-théorie des $\mathrm{C}^{*}-$ algèbres des feuilletages. Cette conjecture a été démontrée par T. Natsume [N2] pour les feuilletages de classe $C^{\infty}$, de codimension un et sans holonomie sur une variété fermée. On propose ici une autre démonstration de la conjecture pour cette classe de feuilletages, plus géométrique et qui s'appuie sur l'isomorphisme de Thom, démontré par A. Connes dans [C3]. L'avantage de cette approche est que le résultat sera valable pour tous les feuilletages de classe $C^{0}$.

La démarche comporte essentiellement trois étapes:

La structure transverse d'un feuilletage est décrite par son pseudogroupe d'holonomie. Pour un feuilletage sans holonomie $(M, \mathcal{F})$, il existe toujours une transversale fermée totale, que l'on peut prendre comme axe d'un bon recouvrement. Par conséquent, le pseudogroupe d'holonomie de $(M, \mathcal{F})$, qui agit sur un axe connexe, est en fait un groupe d'homéomorphismes sans points fixes de $\$^{1}$. Un tel groupe est équivalent (comme pseudogroupe, dans un sens qu'on précisera plus tard) à un groupe d'homéomorphismes sans points fixes de $\mathbf{P}$.

La dynamique topologique de ces groupes décrit la dynamique topologique des feuilietages, ce qui nous donne une première classification en termes d'ensembles minimaux. Cette étude est abordće au paragraphe 1. 
A partir de ces groupes, on construit à la fois:

(1) Le classifiant de $(M, \mathcal{F})$, qui est un feuilletage par plans $\mathcal{F}_{0}$ sur le tore $T^{n}$ de dimension $n$, où $n$ est le rang du groupe d'holonomie Hol $(\mathcal{F})$ de $\mathcal{F}$. Ce feuilletage $\mathcal{F}_{0}$ est défini par une action libre de $\mathrm{R}^{n-1}$. Cette construction est réalisée au paragraphe 3 .

(2) La $C^{*}$-algèbre de $(M, \mathcal{F})$, qui est aussi la $C^{*}$-algèbre de $\left(T^{n}, \mathcal{F}_{0}\right)$, ce qui est fait au paragraphe 2 .

L'isomorphisme de Thom, permet finalement la vérification de la conjecture de Baum-Connes pour les feuilletages considérés.

Je voudrais exprimer ma reconnaissance au Professeur Gilbert Hector pour m'avoir proposé ce travail.

\section{Feuilletages sans holonomie. Étude des sous-groupes sans points fixes de Homéo $+(\mathrm{A})$ et Homéo $+\left(\mathrm{S}^{\mathbb{I}}\right)$}

Considérons la situation ci-dessous:

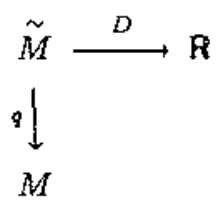

où $q$ est le revêtement universel d'une variété $M$ de groupe fondamental $\pi_{1}(M)$ et $D$ est une fibration (triviale). On définit une action de $\pi_{1}(M)$ sur $\mathbf{R}$ à l'aide d'un homomorphisme:

$$
h: \pi_{1}(M) \longrightarrow \text { Homéo }(\mathbf{R})
$$

Si $D$ est équivariante par rapport à l'action de $\pi_{1}(M)$, elle induit sur $M$ un feuilletage $\mathcal{F}$ de codinension 1 , dont la structure transverse est complètement décrite par le groupe $\operatorname{Imh}=\mathrm{Hol}(\mathcal{F})$. De plus, $\mathcal{F}$ est sans holonomie (c'est à dire, le groupe d'holonornie de toute feuille est trivial) si et seulement si $\mathrm{Hol}(\mathcal{F})$ est sans points fixes (c'est à dire, tout $g \in \mathrm{Hol}(\mathcal{F}), g \neq I d$, est sans point fixe). Bien plus, on a la réciproque suivante ([HH], VIII, théorème 2.2.8):

Théorème 1.1. Tout feuilletage de codimension 1 sans holonomie sur une variété fermée est défni par une fibration équivariante comme ci-dessus.

Ceci nous conduit de façon naturelle à l'étude des sous-groupes sans points fixes de Homéo + ( $R$ ). 
Relation d'ordre partielle sur Homéo+ $(R)$. Sous-groupes sans points fixes.

Soit Homéo + (R) le groupe des homéomorphismes croissants de $\mathbf{R}$. On définit un ordre partiel $\leq$ sur Homéo $+(R)$ par:

$$
g \leq h \text { si } g(x) \leq h(x) \text { pour tout } x \in R .
$$

Un sous-groupe $G$ de Homéo $(R)$, sans points fixes est totalement ordonné et archimédien, donc abélien. Le théorème de Hölder permet de construire un isomorphisme de $G$ dans un sous-groupe de $\mathbf{R}$. Cet isomorphisme est unique à une homothétie près.

Dynamique topologique.

Soit $G \subset$ Homéo $+(R)$. Le sous-groupe $G$ agit sur $\mathbf{R}$ par:

$$
\begin{aligned}
A: G \times \mathbf{R} & \longrightarrow \mathbf{R} \\
(g, x) & \longrightarrow A(g, x)=g(x) .
\end{aligned}
$$

Cette action est effective, et on considère la relation d'équivalence $\rho_{G}$ sur $\mathbf{R}$ dont les classes d'équivalence sont précisément les orbites de l'action $A$, c'est à dire, $G(x)=\{g(x): g \in G\}(x \in \mathbf{R})$. Un ensemble minimal $M$ de $\rho_{G}$ est un ensemble fermé, non vide, saturé pour $\rho_{G}$ et tel qu'aucun sous-ensemble non trivial de $M$ possède les mêmes propriétés; ce qui est équivalent à dire que $\overline{G(x)}=M$ pour tout $x \in M$. En général, $\rho_{G}$ n'admet pas d'ensemble minimal, mais ce sera le cas si $G$ est de type fini.

Lemme 1.2. Si $G \subset H_{0 m e ́ o}(\mathrm{R})$ est de type fini, alors $\rho_{G}$ possède au moins un ensemble minimal.

Preuve: Considérons l'ensemble Sta $(G)=\{x \in \mathbf{R}: g(x)=x$ pour tout $g \in$ $G\}$. Si Sta $(G) \neq \phi$, la conclusion est immédiate. Supposons donc que Sta $(G)=\phi$. Alors, $\lim \inf G(x)=-\infty$ et $\lim \sup G(x)=\infty$ pour tout $x \in \mathrm{R}$. Considérons une famille $\left\{g_{1}, \ldots, g_{n}\right\}$ de générateurs de $G$. Soit $I=[-a, a]\{\alpha\rangle$ $0)$ tel que $g_{i}(0) \in I$ pour tout $i \in\{1, \ldots, n\}$. Alors, $\overline{G(x)} \cap I \neq \phi$ pour tout $x \in \mathbf{R}$. Si on applique le lemme de Zorn aux fermetures des saturés pour $\rho_{G}$ qui coupent $I$, on obtient un ensemble minimal $M_{I}$. Le saturé de $M_{I}$ par $\rho_{G}$ est un ensemble minimal pour $\rho_{G}$.

L'existence d'ensembles minimaux permet une premiere classification des groupes sans points fixes:

Proposition 1.3. Si $G \subset$ Homéo $+(\mathrm{R})$ est sans points fixes, on a les possibilités suivantes:

(1) Si le rang de $G$ est 1, toute orbite est propre fermée, donc un ensemble minimal. 
(2) Si le rang de $G$ est plus grand que $1, \rho_{G}$ possède un ensemble minimal unique $M$. De plus, on a deux cas:

(i) Toutes les orbites sont denses dans $\mathrm{R}$, donc $M=\mathbf{R}$ (on dit alors que $G$ est minimal).

(ii) $M$ est un fermé saturé, sans points isolés et d'intérieur vide. Donc, c'est un Cantor (non compact): on dit que $M$ est un minimal exceptionnel.

Preuve:

(1) C'est trivial.

(2) Supposons que $\rho_{G}$ possède un minimal exceptionnel $M$. Pour $x \in \mathrm{R}-M$, soit $J_{x}=\left(u_{x}, v_{x}\right)$ la composante connexe de $\mathbb{R}-M$ qui contient $x$. Puisque $u_{x} \in M, G\left(u_{2}\right)$ est une orbite non propre car $\overline{G\left(u_{x}\right)}=M$. Comme $u_{x}$ n'est pas isolé dans $M$, il existe une suite $\left\{g_{n}: n \in N\right\} \subset G$ telle que $\lim _{n \rightarrow \infty} g_{n}\left(u_{x}\right)=u_{x}$. Les intervalles $g_{n}\left(J_{x}\right)(n \in \mathrm{N})$ sont deux à deux disjoints, donc $u_{x}=\lim _{n \rightarrow \infty} g_{n}(x) \in \overline{G(x)}$. On déduit que $\overline{G\left(u_{x}\right)} \subset$ $\overline{G(x)}$ et alors $M \subset \overline{G(x)}$. Ceci prouve que $M$ est unique.

\section{Étude des modèles.}

Pour les sous-groupes de Homéo $+(R)$ que nous sommes en train d'étudier, on va voir que l'on peut toujours se ramener à l'étude d'un groupe de translations. Pour cela, on doit d'abord introduire la notion de conjugaison de groupes.

Définition 1.4. Soient $G$ et $G^{\prime}$ deux sous-groupes de Homéo+ $(\mathbf{R})$.

(1) On dit que $G$ est semi-conjugué à $G^{\prime}$, s'il existe une application surjective, croissante et continue $\Psi: R \rightarrow R$ et un homomorphisme surjectif $\varphi: G \rightarrow G^{\prime}$, de façon à avoir le diagramme commutatif suivant:

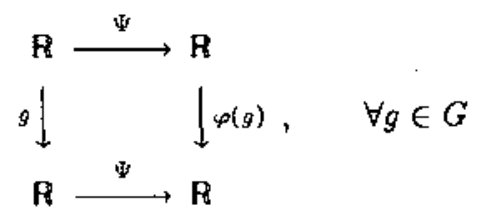

(2) Si $\Psi$ est un honéornorphisme, $\varphi$ es forcément un isomorphisme et on parle alors d'une conjugaison entre les groupes $G$ et $G^{\prime}$.

Remarque. Si $G$ et $G^{\prime}$ sont deux sous-groupes sans points fixes, la propriété archimédienne de $G$ prouve que $\varphi: G \rightarrow G^{\prime}$ est toujours un isomorphisme.

Avec ces définitions, et puisqu'on travaille avec des groupes archimédiens, on obtient le résultat fondamental suivant:

Thèorème 1.5. Si $G$ est un sous-groupe sans points fixes de Homéo $(\mathbf{R})$, on a les possibilités suivantes:

(1) Si le rang de $G$ est $1, G$ est conjugué au groupe des translations entières. 
(2) Dans les autres cas, $G$ est semi-conjugué à un groupe de translations de $\mathbf{R}$. La semi-conjugaison est une conjugaison si et seulement si $G$ est minimal.

Dans le cas $C^{2}$, on a un théorène de type Denjoy-Sacksteder ([HH], VI, théorème 3.10):

Théorème 1.6. Soit $G$ un sous-groupe du groupe Diff ${ }_{+}^{2}(\mathbf{P})$ des difféomorphismes de classe $C^{2}$ croissonts de $\mathrm{R}$, de type fini et tel que $\rho_{G}$ possède un ensemble minimal exceptionnel $M$. Alors, il existe $g \in G$ et $x_{0} \in M$, tels que $g\left(x_{0}\right)=x_{0}$ et $D_{g}\left(x_{0}\right)<1$.

Corollaire 1.7. Si $G \subset D_{i f f_{+}^{2}}(\mathrm{R})$ est de type fini et sans points fixes, alors $G$ est conjugué à un groupe de translations.

Le passage de Homéo $+(\mathbb{A})$ à Homéo+ $\left(S^{l}\right)$.

On peut obtenir Homéo+ $\left(S^{1}\right)$ à partir de Homéo $+(\mathbb{R})$ de la façon suivante:

Considérons $D\left(\$^{1}\right)=\left\{f \in\right.$ Homéo+ $\left._{+}(\mathbf{R}): f(x+1)=f(x)+1\right\}$. Si on désigne par $R \alpha$ la translation de module $\alpha$ dans $\mathbf{R}$ et on considère l'ensemble $C=\left\{R_{k}: k \in \mathbb{Z}\right\}$, on a un isomorphisme Homéo+ $\left(\mathbb{S}^{1}\right) \cong D\left(\mathbb{S}^{1}\right) / C$. Si $q: \mathbb{R} \longrightarrow \$^{1}$ est l'application exponentielle et $f \in D\left(\$^{1}\right)$, on a un diagramme commutatif:

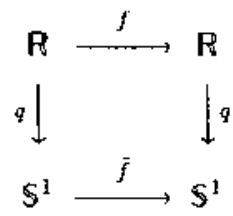

où $\tilde{f} \in$ Homéo $+\left(\boldsymbol{S}^{1}\right)$ est l'application induite par $f$. On appelle $f$ un relèvement de $\bar{f}$. Alors, $\overline{R \alpha}$ est la rotation de $S^{1}$ d'angle $\alpha(\bmod \mathbb{Z})$. Considérons $\bar{G} \subset$ Homéo $_{+}\left(\$^{1}\right)$ et $G$ l'ensemble des relèvements des éléments de $\bar{G}$. On a une suite exacte:

$$
O \longrightarrow \mathbb{Z} \stackrel{k_{1}}{\longrightarrow} G \stackrel{k_{2}}{\longrightarrow} \bar{G} \longrightarrow O
$$

où $h_{1}(k)=R_{k}$ et $h_{2}(f)=\bar{f}$, pour $k \in \mathbb{Z}$ et $f \in G$.

Le théorème 1.5 permet le passage de $G$ a $\bar{G}$, si $G$ est un sous-groupe de Homéo $+(\mathbb{R})$ sans points fixes. Alors, $G$ est un groupe de type fini (de rang $n$ ) et sans points fixes si et seulement si $\bar{G}$ est un groupe de type fini (de rang $n-1$ ) et sans points fixes. En particulier, $\bar{G}$ est abélien. On définit $\rho_{G}$ de façon analogue à $\rho_{G}$. Les orbites de $\rho_{G}$ sont simplement les images par l'application exponentielle des orbites de $\rho_{G}$. On déduit alors de façon evidente les énoncés analogues de la proposition 1.3 et du théorème 1.5 pour $\bar{G}$.

Bien que $G$ et $\bar{G}$ ne soient pas isomorphes comme groupes, la notion d'équivalence de pseudogroupes nous fournit un isomorphisme à un autre niveau, adapté à l'étude des feuilletages: 
Définition 1.8. Deux pseudogroupes de difféomorphismes locaux ([HH], II, définition 2.1.3) $(Q, P)$ et $\left(Q^{\prime}, P^{\prime}\right)$ sont dits équivalents s'il existe une famille $\Phi=\left\{\varphi_{i}\right\}_{i \in I}$ de difféomorphismes $\varphi_{i}$ de domaine $V_{i} \subset Q$ et image $V_{i}^{\prime} \subset Q^{\prime}$, tels que:

(I) $\left\{V_{i}\right\}_{i \in I}$ est un recouvrement ouvert de $Q$.

$\left\{V_{i}^{\prime}\right\}_{i \in I}$ est un recouvrement ouvert de $Q^{\prime}$.

(2) Pour tout $f \in \mathrm{P}$ et tout couple $(i, j)$ tel que $f^{\prime}=\varphi_{j} \circ f \circ \varphi_{i}^{-1}$ soit défini, on a $f^{\prime} \in \mathbf{P}^{\prime}$.

(3) Pour tout $f^{\prime} \in \mathbb{P}^{\prime}$ et tout couple $(i, j)$ tel que $f=\varphi_{j}^{-1} \circ f^{\prime} \circ \varphi_{i}$ soit défini, on a $f \in \mathbf{P}$.

$\Phi$ est appelé un isomorphisme de $\mathbf{P}$ sur $\mathbf{P}^{\prime}$.

L'application exponentielle $q: \mathrm{R} \rightarrow \Phi^{1}$ est un homéomorphisme local, donc elle est localement inversible. Cette observation, et la construction de $\vec{G}$ à partir de $G$, permettent de vérifier aisément le résultat suivant:

Proposition 1.9. L'application exponentielle $q: R \rightarrow S^{1}$ définit une équivalence entre $(\mathcal{R}, G)$ et $\left(\boldsymbol{S}^{3}, \bar{G}\right)$ comme pseudogroupes de difféomorphismes locaux.

Remarque: La proposition 1.3 a un équivalent en termes de feuilletages, obtenu en faisant jouer à la relation d'équivalence définie par $\mathcal{F}$ de rôle joué par $\rho_{G} .([\mathrm{HH}], I V$, section 2.2).

\section{2. $C^{*}$-algèbre et $K$-théorie associées aux sous-groupes sans points fixes de $\mathrm{Homé}_{+}(\mathrm{R})$ et $\mathrm{Homé}_{+}\left(\mathrm{S}^{1}\right)$.}

Soit $\bar{G} \subset$ Homéo $\left(\mathbf{S}^{1}\right)$ sans points fixes et de type fini. Soit $G \subset$ Homéo $+(\mathbb{R})$ le relevé de $\bar{G}$ selon les notations du paragraphe 1. Alors, $G$ est sans points fixes et de type fini. Soit $n$ le rang de $G$. L'action $A$ de $G$ sur $R$ induit une action de $G$ sur $C_{0}(\mathbf{P})$ par *-automorphismes:

$$
\begin{aligned}
A^{*}: G \times C_{0}(\mathrm{P}) & \longrightarrow C_{0}(\mathbb{R}) \\
(g, f) & \longrightarrow A^{*}(g, f): \mathrm{R} \\
& \longrightarrow \mathrm{C} \\
& \longrightarrow A^{*}(g, f)(x)=f\left(g^{-1}(x)\right)
\end{aligned}
$$

Comme $G$ est abélien, donc moyennable, le produit croisé $C_{0}(\mathbf{R}) \times G$ et le produit croisé réduit $C_{0}(\mathrm{R}) \rtimes_{\mathrm{r}} G$ (associés au système dynamique $\left(G, C_{0}(\mathrm{R}), A^{*}\right)$ ) coincident $([\mathrm{P}])$.

Définition 2.1. La $C^{*}$-algèbre $C^{*}(G ; \mathbf{R})=C_{0}(\mathbf{R}) \times G$ est appelée la $C^{*}-$ algebre de groupes de transformation pour l'action de $G$ sur $\mathbf{R}$.

De la mêrne façon, on définit $C^{*}\left(\bar{G} ; \mathbf{S}^{1}\right)=C\left(\mathbf{S}^{1}\right) \times \bar{G}$. La proposition 1.9 , permet d'obtenir le résultat suivant (voir [BGR] et [MRW]): 
Théorème 2.2. Considérons les pseudogroupes de difféomorphismes locaux $(\mathbf{P}, G)$ et $\left(\mathbb{S}^{1}, \bar{G}\right)$. Alors, on a un isomorphisme de $C^{*}$-algèbres:

$$
\left(C_{0}(\mathbf{R}) \rtimes G\right) \otimes k \cong\left(C\left(\mathbb{S}^{\mathrm{I}}\right) \rtimes \bar{G}\right) \otimes k,
$$

où $k$ est l'algèbre des opérateurs compacts sur un espace de Hilbert séparable et de dimension infinie.

Ce résultat réduit l'étude des $C^{*}$-algèbres antéricures à l'étude d'une seule d'entre elles. On va ćtudier les propriétés de $C^{*}\left(\bar{G} ; \$^{1}\right)$.

On va d'abord introduire la notion de tore non commutatif, qui est sans doute l'exemple le plus accessible de variété différentiable non commutative.

Définition 2.3. Le tore non commutatif d'ordre $n$ est la $C^{*}$-algèbre universelle engendrée par $n$ opérateurs unitaires $U_{1}, \ldots, U_{n}$ sur un espace de Hilbert séparable, qui vérifient les conditions de commutativité suivantes:

$u i u j u i^{-1} u j^{-1}=\rho_{i j} \in S^{1}$ pour tout $i, j \in\{1, \ldots, n\}$

Ces $C^{*}$-algèbres possèdent une structure différentiable naturelle, définie par une action ergodique naturelle de $\mathbf{T}^{n}$ comme groupe d'automorphismes ([C2]).

Théorème 2.4. Soit $\bar{G} \subset$ Homéo $\left(S^{1}\right)$ un groupe sans points fixes et de type fini (rang n-1). On a les possibilités suivantes:

(1) Si $n=1$, alors $C^{*}\left(\bar{G} ; \mathbb{S}^{\prime}\right) \cong C\left(\mathbb{S}^{\prime}\right)$.

(2) Si $n>1$, on a encore deux possibilités:

(i) Si $\bar{G}$ est conjugué $\dot{a}$ un groupe de rotations, alors $C^{*}\left(\bar{G} ; \aleph^{1}\right)$ est un tore non commutatif de dimension $n$.

(ii) Si $\bar{G}$ est semn-conjugué $\dot{a}$ un groupe de rotations $\Gamma$, on a une suite exacte:

$$
0 \longrightarrow C^{*}(\bar{G} ; U) \longrightarrow C^{*}\left(\bar{G} ; \$^{1}\right) \longrightarrow C^{*}(\bar{G} ; M) \longrightarrow 0
$$

où $M$ est le minimal exceptionnel de $\rho_{\bar{G}}$ et $U=\mathcal{S}^{1}-M . C^{*}\left(\bar{G}_{j} M\right)$ est un tore non commutatif de dimension $n$ et $C^{*}(\bar{G} ; U)$ est isomorphe a une somme $\oplus_{i \in I} C_{0}(\mathbb{R})$, où $I \subset \mathrm{N}$ (éventuellement fini).

Preuve: Il suffit de voir (2ii). Si $M$ est le minimal exceptionnel pour la relation d'équivalence $\rho_{\bar{G}}, U=\$^{1}-M$ est un ouvert saturé dense dans $\$^{1}$ qui nous fournit un idéal bilatère fermé non trivial $C^{*}(\bar{G}, U)$ de $C^{*}\left(\bar{G} ; \varsigma^{1}\right)$. En plus, on a un homomorphisme surjectif:

$$
C^{*}\left(\bar{G} ; \$^{1}\right) \longrightarrow C^{*}(\bar{G} ; M)
$$

dont le noyau est justement $C^{*}\left(\bar{C}_{r} ; U\right)([\mathbf{G}])$. On a donc une suite exacte de $C^{\star}$-algèbres:

$$
0 \longrightarrow C^{*}(\bar{G} ; U) \longrightarrow C^{*}\left(\bar{G} ; \mathbb{S}^{1}\right) \longrightarrow C^{*}(\bar{G} ; M) \rightarrow 0
$$


En outre, $\bar{G}$ agit sur $M$ comme groupe de rotations $\Gamma$, donc $C^{*}(\bar{G} ; M)$ est un tore non commutatif de dimension $n$. Soit $J$ une composante connexe de $U$. L'action de $\bar{G}$ sur $J$ est triviale. Soit Sat $(J)=\underset{\bar{g} \in \bar{G}}{\bigcup} \bar{g}(J)$; l'action de $\bar{G}$ sur Sat $(J)$ est libre et propre et $J \cong$ Sat $(J) / \vec{G}$. Donc, $U=\bigcup_{i \in I} V_{i}$, où les $V_{i}=$ Sat $\left(J_{i}\right)\left(J_{i}\right.$ est une composante connexe de $\left.U\right)$ sont des ouverts saturés deux à deux disjoints et $I \subset \mathrm{N}$ est éventuellement fini. $\mathrm{On}$ a alors les isomorphismes suivants:

$$
\begin{aligned}
C^{*}\left(\bar{G} ; V_{i}\right) & \cong C_{0}\left(V_{i} / \bar{G}\right) \otimes k\left(L^{2}(\bar{G})\right) \cong C_{0}\left(J_{i}\right) \otimes k\left(L^{2}(\bar{G})\right) \cong \\
& \cong C_{0}(\mathrm{R}) \otimes k\left(L^{2}(\bar{G})\right) .
\end{aligned}
$$

On peut donc décrire la $C^{*}$-algèbre $C^{*}(\bar{G} ; U)$ par:

$$
C^{*}(\bar{G} ; U) \cong \underset{i \in I}{\oplus} C^{*}\left(\bar{G} ; V_{i}\right) \cong \underset{, \in I}{\oplus}\left(C_{0}(\mathbf{R}) \otimes k\left(L^{2}(\bar{G})\right)\right.
$$

Remarque. La minimalité de $\bar{G}$ équivaut justement à la non-existence d'idéaux bilatères fermés pour $C^{*}\left(\bar{G} ; \$^{3}\right)$.

Puisqu'un tore non commutatif est un produit croisé itéré d'actions de $\mathbf{Z}$, on peut utiliser la suite exacte de Pimsner-Voiculescu [PV] en plusieurs étapes, et on déduit que les $K$-groupes des tores non commutatifs sont les mêmes que ceux d'un tore ordinaire de la même dimension. On obtient alors le corollaire suivant:

Corollaire 2.5. Dans les conditions du théorème 2.4 (2i), on a un isomorphisme en $K$-théorie:

$$
K_{*}\left(C^{*}\left(\bar{G} ; S^{1}\right)\right) \cong K^{*}\left(T^{n}\right)
$$

où $K_{*}$ signifie $K$-théorie pour algèbres d'opérateurs (/C2/) et $K^{*} K$-théorie topologique à supports compacts.

Corollaire 2.6. Dans les conditions du théorème 2.4 (2ii), on obtient la suite exacte en $K$-théorie suivente:

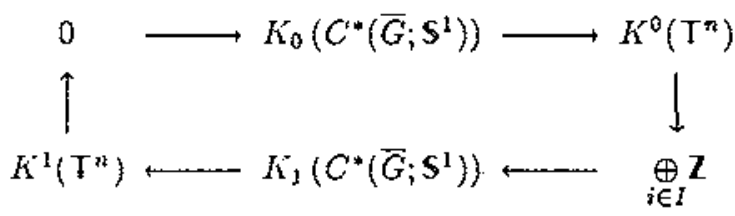

Preuve: Il suffit d'utiliser la suite exacte du théorème 2.4 (2ii), qui passe à une suite exacte de six-termes en $K$-théorie [C2], le corollaire 2.5 et les propriétés suivantes des $K$-groupes:

(1) $K_{*}\left(\underset{i \in I}{\oplus} A_{i}\right) \cong \bigoplus_{i \in I} K_{*}(A i)$ pour tout famille de $C^{*}$-algèbres $\{A i: i \in I\}$.

(2) $K_{*}(A \otimes k) \cong K_{*}(A)$ pour toute $C^{*}$-algèbre $A$.

(3) $K_{0}\left(C_{0}(\mathrm{R})\right) \cong K^{0}(\mathrm{P}) \cong 0$

$K_{\mathrm{I}}\left(C_{0}(\mathrm{R})\right) \cong K^{1}(\mathrm{R}) \cong \mathrm{Z}$. 


\section{Feuilletages classifiants des feuilletages sans holonomie}

Suspension de $G$ et de $\bar{G}$.

Soient $\bar{G} \subset$ Homéo $+\left(S^{3}\right)$ et $G \subset$ Homéo $(R)$ des groupes sans points fixes et de type fini, selon les notations du paragraphe 1 . Solent $n$ le rang de $G,\left\{g_{1}, \ldots, g_{n}\right\}$ une famille de générateurs et $A$ l'action de $G$ sur $R$. On définit une action libre et proprement discontinue de $G$ sur $R^{n} \times \mathbf{R}$ par:

$$
\begin{aligned}
A_{0}: G \times\left(\mathrm{R}^{n} \times \mathrm{R}\right) & \longrightarrow \mathrm{R}^{n} \times \mathrm{R} \\
\left(g, x_{3}, \ldots, x_{n}, x\right) & \longrightarrow\left(x_{1}+k_{1}, \ldots, x_{n}+k_{n}, g(x)\right),
\end{aligned}
$$

où $g=g_{1}^{k_{1}} \circ \cdots \circ g_{n}^{k_{n}}$. On obtient un diagramme commutatif:

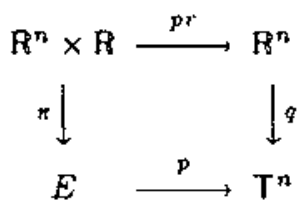

où $p r$ est la projection canonique, $\pi$ est l'application quotient par $A_{0}, q$ est le revêtement universel, $E$ est l'espace quotient et $p$ est l'application induite par $p r$. Cette construction est appelée la suspension de $G$. On a en plus le résultat suivant:

Proposition 3.1. Dans le diagramme (1):

(1) E est difféomorphe $\dot{a} \mathrm{I}^{n} \times \mathbf{R}$,

(2) Il est muni d'une structure plate non triviale,

(3) Les nappes horizontales de cette structure sont les feuilles d'un feuille. iage par plans $\mathcal{F}_{G}$ sur $E$ défini par une action libre de $\mathbf{R}^{n}$.

Preuve: Le feuilletage horizontal sur $\mathrm{R}^{n} \times \mathrm{R}$ est défini par une action libre de $\mathrm{R}^{n}$, qui passe au quotient en une action de $\mathrm{R}^{n}$ sur $E$ induisant le feuilietage $\mathcal{F}_{G}$. Pour le reste de la proposition, on peut consulter $[\mathbf{H H}]$, II, proposition 1.2.2.

Pour $\bar{G} \subset \mathrm{Homé}_{+}\left(\mathbf{S}^{\overline{1}}\right)$ on realise la construction analogue, qui fournit un diagramme commutatif:

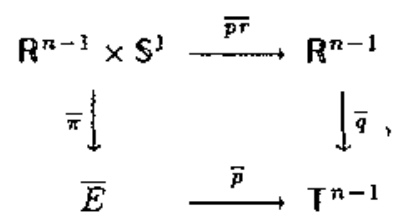

où $\bar{E}$ est difféomorphe à $\mathrm{T}^{n} \cdot \bar{E} \stackrel{\bar{p}}{\longrightarrow}{T^{n-1}}^{n-1}$ est un fibré plat de fibre $S^{1}$. Les nappes horizontales de cette structure sont les feuilles d'un feuilletage par plans 
$\mathcal{F}_{\bar{G}}$ sur $\bar{E}$, défini par une action libre de $\mathrm{R}^{n-1}$. Bien plus, on a une section canonique $\sigma: \bar{E} \longrightarrow E$ définie de la façon suivante:

Considérons le diagramme commutatif:

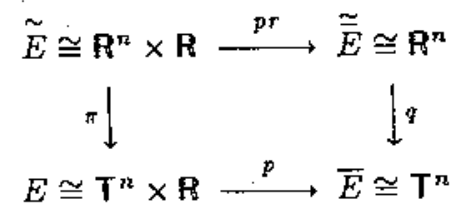

Le feuilletage $\mathcal{F}_{\bar{G}}$ sur $T^{n}$ se relève à un feuilletage par plans $\tilde{\mathcal{F}}_{\bar{G}}$ sur $R^{n}$, qui est défini par une fibration localement triviale de fibre $\mathbf{R}^{n-1}$ et base $\mathbf{R}, \mathbf{R}^{n} \stackrel{D}{\longrightarrow} \mathbf{R}$. On définit la section $\tilde{\sigma}: \mathbf{R}^{n} \rightarrow \mathbf{R}^{n} \times \mathbf{R}$ par $\tilde{\sigma}(z)=(z, D(z))$ pour tout $z \in \mathbf{R}^{n}$. Cette application passe au quotient en une section $\sigma: \mathbf{T}^{n} \rightarrow \mathbf{T}^{n} \times \mathbf{R}$. La transversalité de $\sigma$ permet de conclure qu'il existe un feuilhetage unique de codimension $1, \mathcal{F}=\sigma^{*}\left(\mathcal{F}_{G}\right)$ sur $T^{n}$, de façon à avoir un homomorphisme de feutilletages $\sigma:\left(\mathbf{T}^{n}, \mathcal{F}\right) \longrightarrow\left(\mathbf{T}^{n} \times \mathbb{R}, \mathcal{F}_{G}\right)$. Par construction, ces deux feuilletages possèdent des pseudogroupes d'holonomie èquivalents $\left(\mathbf{S}^{\mathbb{I}}, \bar{G}\right)$ et $(\mathbf{R}, G)$.

\section{Feuilletages sans holonomie et feuilletages par plans.}

On a donc démontré le résultat suivant:

Théorène 3.2. Si $\bar{G} \subset$ Homéo $_{+}\left(S^{1}\right)$ est un groupe sans points fixes et de type fini (de rang $n-1$ ), il existe un feuilletage par plans sur $\mathrm{T}^{n}$, dont le pseudogroupe d'holonomie est $\left(\boldsymbol{S}^{1}, \bar{G}\right)$, et il est unique à conjugaison près.

L'idée d'associer une $C^{*}$-algèbre non commutative à une variété feuilletée est due à $A$. Connes $([\mathrm{C} 1]$ et $[\mathrm{C} 2])$. Cette $C^{*}$-algèbre représente l'espace des feuilles et se construit à partir du groupoïde d'holonomie du feuilletage. Pour la varieté feuilletée $\left(T^{n}, \mathcal{F}_{\bar{G}}\right)$, le calcul de la $C^{*}$-algèbre est une conséquence inmédiate de la caractérisation des $C^{*}$-algèbres pour les feuilletages définis par des actions de groupes de Lie $([\mathrm{C} 2])$ :

Proposition 3.3. Soit $\left(\Phi^{n}, \mathcal{F}_{\bar{G}}\right)$ le feuilletage construit ci-dessus. Alors, on a un isomorphisme de $C^{*}$-algébres:

$$
C^{*}\left(\mathbf{T}^{n}, \mathcal{F}_{\bar{G}}\right) \cong C\left(\mathbf{T}^{n}\right) \rtimes \mathbf{R}^{n-1} .
$$

Remarque: Le théorène 3.2 établit, d'une autre part, l'isomorphisme de $C^{*}$-algèbres suivant $([\mathbf{R}])$ :

$$
C^{*}\left(\mathbf{T}^{n}, \mathcal{F}_{\bar{G}}\right) \cong C^{*}\left(\bar{G} ; \mathbb{S}^{1}\right) \otimes k
$$

La $K$-théorie associée à $C^{*}\left(T^{n}, \mathcal{F}_{\bar{G}}\right)$ se déduit comme un corollaire du résultat suivant de A. Connes ([C3]): 
Lemme 3.4. Si $(A, H, a)$ est un système dynamique, où $H$ est un groupe de Lie résoluble et simplement connexe et $j \in \mathrm{Z} / 2$ est la dimension de $H$ module 2 , alors on a un isomorphisme naturel

$$
K_{i}\left(A \rtimes_{\alpha} H\right) \cong K_{i+j}(A),
$$

appelé l'isomorphisme de Thom.

Si on applique ce lemme au système dynamique $\left(C\left(\mathrm{~T}^{n}\right), \mathrm{R}^{n-1}, \alpha\right)$ (où $\alpha$ est l'action libre de $\mathrm{R}^{n-1}$ qui induit le feuilletage $\mathcal{F}_{\vec{G}}$ ) - compte tenu de l'isomorphisme en $K^{\prime}$-théorie $K_{*}\left(C_{0}(X)\right) \cong K^{*}(X)$ pour les espaces localement compacts $X-$, on obtient le résultat suivant:

Proposition 3.5. Si $j+1 \in Z / 2$ est $n$ module 2 , on a l'isomorphisme naturel de Thom:

$$
K_{i}\left(C^{*}\left(T^{n}, \mathcal{F}_{\bar{G}}\right)\right) \cong K^{r+j}\left(\mathrm{~T}^{n}\right) .
$$

Finissons cette section en reliant les feuilletages sans holonomie et les feuilletages par plans sur des tores que l'on vient de construire. On va introduire d'abord la notion de feuilletage classifant d'un feuilletage donné:

Défnition 3.6. Soit $(M, \mathcal{F})$ une variété feuilletée. On dit que $\left(M_{0}, \mathcal{F}_{0}\right)$ est un ferilletage classifiant $\operatorname{de}(M, \mathcal{F})$, si on a les propriétés suivantes:

(1) Les pseudogroupes d'holonomie $(\mathrm{Q}, \mathrm{P})$ et $\left(\mathrm{Q}_{0}, \mathrm{P}_{0}\right)$ de $(M, \mathcal{F})$ et $\left(M_{0}, \mathcal{F}_{0}\right)$ respectivement sont équivalents.

(2) Pour toute feuille $L_{0} \in \mathcal{F}_{0}$, la représentation d'holonomie hol : $\pi_{1}\left(L_{0}\right) \rightarrow$ hol $\left(L_{0}\right)$ est injective.

(3) Le revêtement d'holonomie de toute feuille de $\mathcal{F}_{0}$ est contractile.

Considérons un feuilletage de codimension 1 et sans holonomie $\mathcal{F}$ sur une variété fermée $M$. Le théorème 1.1 fournit un groupe $G=\mathrm{Hol}(\mathcal{F})$ sans points fixes et de type fini. Si on considère $\bar{G}$ avec les notations du paragraphe 1 , on obtient le résultat suivant:

Proposition 3.7. Dans les conditions imposées, le pseudogroupe d'holonomie de $(M, \mathcal{F})$ est équivalent à $\left(\mathbf{S}^{1}, \bar{G}\right)$.

Alors, on déduit de façon immédiate le résultat clé de ce paragraphe:

Théorème 3.8. Dans les conditions antérieures, sin est le rang du groupe d'holonomie Hol $(\mathcal{F})$ de $(M, \mathcal{F})$, il existe un feuilletage par plans sur $\mathrm{T}^{n}$ qui est un feuilletage classifiant pour $(M, \mathcal{F})$.

On a tous les outils nécessaires pour vérifier la conjecture de Baum-Connes pour les feuilletages sans holonomie.

Cette conjecture s'énonce dans les termes suivants: 
Pour une variété feuilletée $(M, \mathcal{F})$ l'espace des feuilles est un espace complètement intraitable en général. Il résulte alors un problème définir la $K$-théorie pour un tel espace singulier. A. Connes $([\mathrm{C} 2])$ définit:

(1) Une $K$-théorie analytique pour l'espace des feuilles, $K_{*}\left(C^{*}(M, \mathcal{F})\right.$ ), qui utilise la $C^{*}$-algèbre $C^{*}(M, \mathcal{F})$ associée à $\mathcal{F}$.

(2) Une $K$-théorie topologique pour l'espace des feuilles, $K_{*, r}(B G)$, qui se définit comme une certaine $K$-homologie tordue de l'espace classifiant du groupoïde d'holonomie du feuilletage $\mathcal{F}$.

A. Connes signale l'existence d'une application naturelle:

$$
\mu: K_{*, \tau}(B G) \longrightarrow K_{*}\left(C^{*}(M, \mathcal{F})\right),
$$

et il conjecture que $\mu$ est toujours un isomorphisme (quand les groupes d'holonomie n'ont pas de torsion).

Dans le cas particulier des feuilletages sans holonomie, cette conjecture s'énonce de la façon suivante:

Conjecture de Baum-Connes. Soit $(M, \mathcal{F})$ une variété feuilletée et $\left(M_{0}, \mathcal{F}_{0}\right)$ un feuilletage classifiant de $(M, \mathcal{F})$. On a un isomorphisme naturel:

$$
\mu: K^{*}\left(M_{0}\right) \longrightarrow K_{*}\left(C^{*}(M, \mathcal{F})\right)
$$

Donc, dans nos hypothèses, la conjecture est vérifiée comme une conséquence immédiate de la proposition 3.5 et du théorème 3.8 :

Théorème 3.9. Si $(M, \mathcal{F})$ est un feuilletage sans holonomie et de codimension 1 sur une variété fermée $M$, la conjecture de Baum-Connes est vraie.

\section{References}

[BGR] L.G.Brown, P.Green AND M.A.RIeffel, Stable isomorphism and strong Morita equivalence of $C^{*}$-algebras, Pacific $J$. Math. 71 (1977), 349-363.

[C1] A.CONNES, Sur la théorie non commutative de l'intégration, Lecture Notes in Maths 725 (1979), Springer-Verlag, Berlin, New York, Heidelberg.

[C2] A.CONnes, A survey of foliations and operator algebras, Proc. Symp. Pure Math. 38 (1982), 521-628.

[C3] A.CONNES, An analogue of the Thom isomorphism for cross-products of a $C^{*}$-algebra by an action of R, Adv. in Math. 39 (1987), $31-55$.

[G] P.GREEN, $C^{*}$-algebras and transformation groups with smooth orbit space, Pacific J. Math. 72 (1977), 71 97. 
[HH] G.HECTOR AND U.HIRSCH, Introduction to the Geometry of Foliations (Part A and B), Friedr. Vieweg and Sohn, Braunschweig, 1986-1987.

[MRW] P.S.Mulhy, J.N.Renault and D.P.Williams, Equivalence and isomorphism for groupoid $C^{*}$-algebras, $J$. Operator Theory 17 (1987), 3-22.

[N1] T.NATSUME, The $C^{*}$-algebra of codimension one foliation without holonomy, Math. Scand. 56 (1985), 96-104.

[N2] T.NATSUME, Topological $K$-theory for codimension one foliation without holonomy, Adv. Stud. Pure Math. 5 (1985), 15-27, North-Holland, Amsterdam-New York.

[P] G.K.PEDERSEN, $C^{*}$-algebras and their automorphism groups, London Math. Soc., Monographs 14, Academic Press (1979).

[PV] M.PIMSNER AND D.VOICULESCU, Exact sequences for $K$-groups and Ext-groups of certain cross-product $C^{*}$-algebras, $J$. Operator Theory 4 (1980), 93-118.

[R] J.N.Renault, $C^{*}$-algebras of groupoids and foliations, Proc. Symp. Pure Maths. 88 (1982), 339-350.

\footnotetext{
Departamento de Matemáticas

Facultad de Ciencias

Universidad del Pais Vasco-Euskal Herriko Unibertsitatea

Apartado 644-48080 Bilbao,SPAIN
}

Rebut el 16 de Maig de 1989 